\title{
Aerogeles con aplicaciones en biomedicina y medioambiente
}

\author{
J. A. TOLEDO-FERNÁNDEZ, R. MENDOZA-SERNA, V. MORALES-FLÓREZ, N. DE LA ROSA-FOX, A. SANTOS ${ }^{\circ}$, \\ M. PIÑERO ${ }^{x}$, L. ESQUIVIAS \\ Departamento de Física de la Materia Condensada, Facultad de Ciencias, \\ 'Departamento de Cristalografía y Mineralogía, CASEM \\ xDepartamento de Física Aplicada, CASEM \\ Universidad de Cádiz \\ C/ República Saharaui, s/n. 11510 Puerto Real, España.
}

\begin{abstract}
Es posible preparar materiales híbridos inorgánico-orgánicos incorporando una fase orgánica sobre un sol obtenido por hidrólisis de metalorgánico mientras se aplican ultrasonidos de alta potencia. Cuando las dos fases quedan químicamente enlazadas resulta un sono-ormosil (de ORganic MOdified SILicate) conocido también como ormosil duro. Unas de las aplicaciones de estos materiales atañe el dominio de la biotecnología pues llegan a ser bioactivos cuando contienen Ca, cumpliendo con ello el primer requisito para su validez como implantes óseos. La fuente de calcio, seleccionada para optimizar el proceso de secado supercrítico en etanol, ha sido partículas de wollastonita, $\left(\mathrm{CaSiO}_{3}\right)$, material bioactivo, lo que al influir sobre el volumen poroso y el radio de los poros, permite controlar la densidad y situar la resistencia mecánica en el intervalo de valores propios de los huesos humanos esponjosos. Por otra parte, los poros pueden hacerse químicamente activos y usarse como soportes estructurales para la captación y fijación de gases.
\end{abstract}

Palabras clave: Sol-gel, materiales hibridos orgánico-inorgánico, aerogeles bioactivos, captación de gases.

\section{Aerogels with applications in biomedicine and environment}

It is possible to synthesise inorganic-organic hybrid materials incorporating the organic phase to a sol prepared from a metal organic under high power ultrasounds. When both phases are chemically bonded a sono-ormosil (from ORganic MOdified SILicate) results, also known as hard ormosil. One of the applications of these materials concerns the biotechnologies since they become bioactive when are doped with $\mathrm{Ca}$, fulfilling this way the preliminary condition to be considered for bone implants. The addition of silicate particles allows modifying the pore volume and radius. We have used these gels with particles, as precursor of the bioactive component, in order to act on the porosity for controlling the density and adequate the mechanical strength to that of the human cancellous bone. In like manner, the pores becomes chemically active and be used as structural support for noxious atmospheric gas sequestration.

Keywords: Sol-gel, hybrid organic-inorganic materials, bioactive aerogels, gases sequesters.

\section{INTRODUCCIÓN}

Uno de los principales problemas en la preparación de materiales masivos a partir de geles es evitar la rotura del gel durante el secado causada por las fuerzas capilares asociadas con la interfaz gas-líquido. La forma más eficaz de neutralizar los efectos indeseados de la tensión superficial es tratar al gel en una autoclave en condiciones supercríticas de presión y temperatura para el disolvente, cuidando de que el camino del tratamiento térmico no cruce la curva de equilibrio líquidovapor. Con esta técnica, inicialmente desarrollada por Kistler (1), se producen los aerogeles.

Los aerogeles de sílice son materiales nanoestructurados, transparentes, químicamente inertes, y altamente porosos. $\mathrm{Su}$ densidad puede variar desde $0.0019 \mathrm{~g} \mathrm{~cm}^{-3}$ (2) hasta $0.9 \mathrm{~g} \mathrm{~cm}^{-3}$, con una alta porosidad, próxima al $90 \%$, con poros de tamaño de orden nanométrico que dan lugar a superficies específicas entre 600 y $1000 \mathrm{~m}^{2} \mathrm{~g}^{-1}$; su composición se mantiene idéntica a la de la sílice vítrea $(3,4)$. Sus principales limitaciones son su fragilidad y su alta higroscopicidad.
Una propuesta para superar estos inconvenientes consiste en disponer de materiales híbridos orgánicos-inorgánicos (MHOI) mediante la inclusión de polímeros orgánicos hidrófobos que, modificando la red polimérica de sílice, aumentan la elasticidad y resistencia a la fractura del material final. Hemos modificado el proceso estándar de preparación de geles por hidrólisis y polimerización de compuestos metal-orgánicos, mediante la aplicación de ultrasonidos de alta potencia que, al evitar el uso de disolvente común del metal-orgánico y el agua, da lugar a materiales con genuinas propiedades, conocidos como sonogeles (5). La cavitación ultrasonora crea un entorno único para las reacciones sol-gel que dan características muy particulares a los geles resultantes: alta densidad, fina textura y estructura homogénea. Si la fase orgánica se incorpora en el sol inorgánico precursor asistiendo el proceso con ultrasonidos y ambas fases quedan químicamente enlazadas tras la gelificación se forma un sonoormosil, también conocido como ormosil duro. En este tipo 
de material, el polímero orgánico suministra flexibilidad, baja densidad, tenacidad y deformabilidad, mientras que la parte inorgánica contribuye con la dureza de la superficie, resistencia mecánica, transparencia y alto índice de refracción. Así estos materiales combinan las ventajas del procesado sol-gel en cuanto al uso de precursores metal-orgánicos, disolventes orgánicos y baja temperatura de proceso, con las características específicas de los polímeros orgánicos como componentes hidrofóbicas/hidrofílicas.

Estos materiales son tolerados por el organismo humano pues se crea un tejido fibroso cuando se implantan en el cuerpo, pero para que puedan unirse al hueso deben ser bioactivos. En tal caso, crece una capa de de carbonato de hidroxiapatita (CHA) que envuelve el material cuando se inmergen en plasma sanguíneo o, in vitro, si se inmerge en una solución acuosa acelular que contiene una concentración estandarizada de iones inorgánicos casi igual a la del plasma humano (SBF, Simulated Body Fluid), propuesta por Kokubo et al. (6). Este es un experimento común en el desarrollo de nuevos biomateriales, para el seguimiento de la formación de $\mathrm{CHA}$ (7). Además, para que rindan lo que se les pide, se requiere que estos materiales aproximen su comportamiento mecánico al de los huesos humanos.

Aparece bioactividad cuando se incluye calcio en el sol inicial. Si el $\mathrm{Ca}$ (II) se añade desde una sal, sucede que se va la mayor parte de él durante el secado, arrastrado por el propio disolvente. Como alternativa lo hemos añadido en forma de partículas de wollastonita $\left(\mathrm{CaSiO}_{3}\right)(8)$, con el doble propósito de evitar la eliminación de $\mathrm{Ca}(\mathrm{II})$ y modificar el comportamiento mecánico del gel.

Por otra parte, los aerogeles inorgánicos pueden ser usados para la adsorción, almacenamiento y fijación de gases contaminantes. Si se añade durante el procesado una fase activa, se puede conseguir que los aerogeles de base silícea sean químicamente activos y puedan usarse como soportes estructurales para diversas catálisis. Asílo demuestran trabajos recientes $(9,10,11)$, en los que una mezcla de gases puede llegar a fluir poro a poro y eventualmente a través de todo el gel, de tal forma que los gases quedan adsorbidos y pueden eliminarse a partir de una reacción posterior en función de su actividad química. Finalmente, en nuestro estudio esa fase activa consiste en partículas de wollastonita, que reproducen miméticamente la reacción de carbonatación que tiene lugar en procesos naturales (12) al entrar en contacto con $\mathrm{CO}_{2}, \mathrm{y}$ aprovecha la eficiencia de los aerogeles basada en su alta porosidad y alta superficie específica.

\section{MÉTODO EXPERIMENTAL}

Hemos preparado los geles híbridos a partir de tetraetoxisilano (TEOS), como precursor de la fase inorgánica, y PDMS (polidimetilsiloxano, DMS12, peso molecular promedio 400-700 g/mol) para la fase orgánica, siguiendo un procedimiento que ya hemos descrito (13).

Por otra parte, hemos precipitado partículas de $\mathrm{CaSiO}_{3}$ (wollastonita) por hidrólisis controlada de TEOS y nitrato de calcio tetrahidratado $\mathrm{Ca}\left(\mathrm{NO}_{3}\right) \cdot 4 \mathrm{H}_{2} \mathrm{O}$ según un proceso análogo al que usamos para la precipitación de partículas de zirconia dopada con itrio (14). El nitrato de calcio y el TEOS se disolvieron en una solución de etanol, hasta una concentración a $0.2 \mathrm{~mol} / 1$. Tras dos horas de agitación magnética se añade $0.33 \mathrm{~mol} / 1$ de $\mathrm{NaOH}$ a la solución anterior manteniendo la agitación. Entonces se obtiene un precipitado de silicato cálcico amorfo que se extrae por centrifugado. Para su lavado, el precipitado se dispersa en agua destilada y se le somete a la acción de un transductor de ultrasonidos de 20 $\mathrm{kHz}$ durante 1 minuto, tras lo cual se vuelve a centrifugar. Este proceso se repite varias veces. Los polvos se dejan secar en una estufa a $100^{\circ} \mathrm{C}$ durante $12 \mathrm{~h}$. Luego los polvos se calcinan durante $2 \mathrm{~h}$ a $500^{\circ} \mathrm{C}$ subiendo la temperatura $3^{\circ} \mathrm{C} / \mathrm{min}$. A $500^{\circ} \mathrm{C}$ permanece durante $120 \mathrm{~min}$. La bajada de temperatura es también de $3^{\circ} \mathrm{C} / \mathrm{min}$. Así se obtiene polvos de wollastonita amorfa. El tamaño de las partículas sintetizadas es de algunas decenas de nanómetros. Este aspecto fue examinado mediante microscopía electrónica de barrido (Figura 1) y la distribución de tamaño de poros obtenida mediante porosimetría de $\mathrm{Hg}$ (recuadro en la Figura 1) confirman el resultado, pues la distribución presenta primero un pico agudo entre 3 y $5 \mathrm{~nm}$ de radio y la segunda parte de la curva está formada por un máximo en torno a $10 \mathrm{~nm}$ de radio, seguido de una larga cola que corresponde a una distribución de agregados.

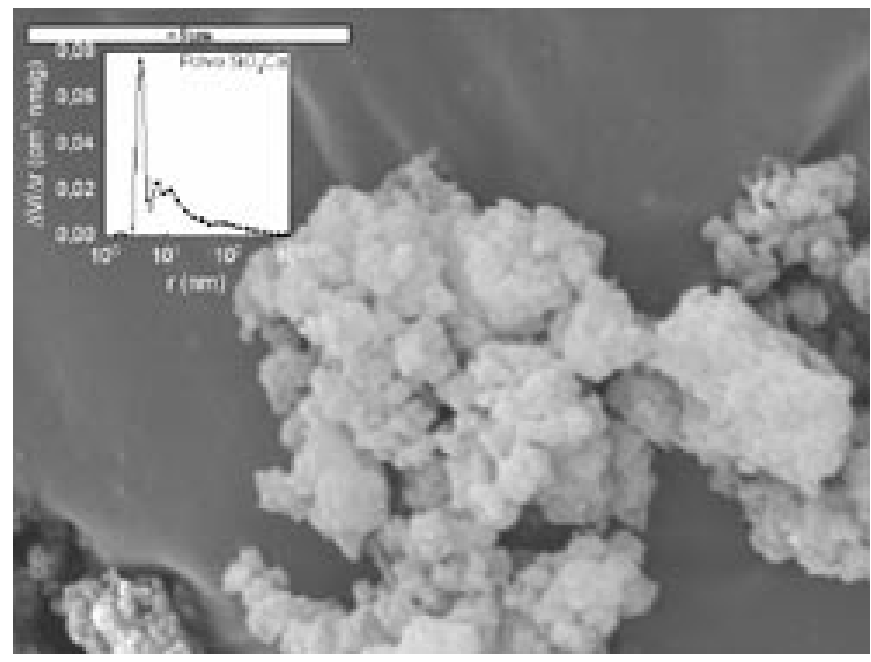

Fig. 1- Micrografía de microscopía electrónica de barrido de los polvos de wollastonita tras tratamiento térmico a $500^{\circ} \mathrm{C}$. En el recuadro está representada la distribución de tamaño de poros de estos polvos obtenida por porosimetría de intrusión de Hg. La línea sólo sirve de guía para los ojos.

Una vez secos, los polvos se dispersan en $4 \mathrm{ml}$ de $\mathrm{EtOH}$ agitando la mezcla durante $3 \mathrm{~min}$ con ultrasonidos y se añaden al sol recién preparado mientras se le aplica la dosis de U.S. precisa para que el tiempo de gelificación sea inferior al minuto. Para las pruebas de bioactividad se prepararon pastillas de wollastonita pura, tratada a $500{ }^{\circ} \mathrm{C}$ (amorfa), y a $1000{ }^{\circ} \mathrm{C}$ (cristalina) y compuestos aerogel/ wollastonita con un $15 \%$ en peso de contenido nominal de wollastonita. Después se deja en la estufa a $50^{\circ} \mathrm{C}$ durante 1 día, tras lo cual se extrae el gel del molde cilíndrico y se deja envejecer una semana en un recipiente cerrado con exceso de etanol con el fin de disolver las trazas de agua en el interior de los poros del gel húmedo. Seguidamente la muestra se coloca en la autoclave con la cantidad suficiente de etanol para alcanzar las condiciones supercríticas del mismo $\left(243^{\circ} \mathrm{C}\right.$ y $\left.63 \mathrm{~atm}\right)$. Dichas condiciones se alcanzan mediante un lento calentamiento $\left(1^{\circ} \mathrm{C} / \mathrm{min}\right)$ con el fin de minimizar la mayor expansión térmica del etanol respecto del gel de sílice. La evaporación del volumen adicional de etanol permite alcanzar las condiciones supercríticas sin 
cruzar en ningún momento la curva de equilibrio líquidovapor evitándose así tensiones capilares y la rotura del gel. Una vez estabilizada la autoclave en condiciones supercríticas, se comienza la evacuación del disolvente gota a gota, proceso en el que se emplea 150 minutos, aproximadamente, tras lo cual se deja enfriar lentamente sin asistencia. Al día siguiente se abre la autoclave y se extraen las muestras secas. Las muestras se obtuvieron en forma cilíndrica de altura $18 \mathrm{~mm}$ y diámetro $8 \mathrm{~mm}$.

El código que utilizaremos de las muestras será PDMSX, donde la $\mathrm{X}$ indicará el tanto por ciento en peso de la fase orgánica en la matriz del gel de TEOS respecto al óxido silicio total. Uno de los efectos de los ultrasonidos es evitar la ciclidación del PDMS, por ello se añade al TEOS previamente hidrolizado en condiciones subestequiométricas de agua. La integración de las cadenas del polímero en la estructura del aerogel puede apreciarse por microscopía electrónica de alta resolución (13). Como consecuencia, los tiempos de gelificación se acortan. Los análisis termogravimétricos (TGA) de estos aerogeles muestran la retención de los grupos orgánicos (15).

La microscopía electrónica de transmisión se realizó en un equipo de $400 \mathrm{keV}$ en el Servicio Central de Ciencia y Tecnología de la Universidad de Cádiz. Para la observación, los aerogeles pulverizados se dispersan en un disolvente y mediante la inmersión de una rejilla con carbón agujereado y su posterior secado se obtiene la muestra lista para su observación.

Se obtuvieron las isotermas de adsorción de $\mathrm{N}_{2}$ a $77 \mathrm{~K}$ en función de la presión de vapor relativa a la de saturación del nitrógeno líquido tras desgasificar la muestra durante 2 horas a $100^{\circ} \mathrm{C}$ y $0.01 \mathrm{~Pa}$. Se analizaron las representaciones del volumen adsorbido en función del espesor estadístico en la formación de multicapas con el fin de analizar la microporosidad de estos sistemas. Dicha microporosidad se evaluó a partir del modelo de Dubinin-Radushkevich (DR) (16), la mesoporosidad fue estimada a partir de los datos de superficie específica calculada por el clásico método de BET. Permitiendo de esta forma conocer la contribución de ambos rangos de poros. En este sentido se calculó las distribuciones de tamaños de mesoporos a partir del método BJH (17) y los valores medios se comparan con el diámetro hidráulico calculado a partir de la superficie específica y volumen poroso totales resultado de la suma DR+BET.

Los ensayos mecánicos de compresión uniaxial se realizaron en un aparato equipado con una celda de carga de $5 \mathrm{kN}$, a una velocidad de carga constante de $0.5 \mathrm{~mm} / \mathrm{s}$.

Para el control de la bioactividad in vitro, tras la inmersión de las muestras en $40 \mathrm{~mL}$ de $\mathrm{SBF}$ a $37^{\circ} \mathrm{C}$ por periodos de siete días, se apartaron de la solución, se enjuagaron abundantemente con agua y acetona y, finalmente, secaron a temperatura ambiente. Luego se observaron mediante microscopía electrónica de barrido y espectroscopía de infrarrojos para detectar la presencia de $\mathrm{CHA}$.

Para los experimentos para la fijación del $\mathrm{CO}_{2}$ se utilizaron tres tipos de muestras: polvo sintético de wollastonita pura, y dos aerogeles de sílice con polvos de wollastonita. El contenido en $\mathrm{CaO}$ de estos materiales compuestos lo evaluamos mediante fluorescencia de rayos $x$, resultando $9 \%$ y un $26 \%$ en peso, respectivamente. La primera de estas tres muestras se justifica para probar la capacidad de la wollastonita como agente fijador del gas, antes de integrarlo en la matriz del aerogel. La captación de $\mathrm{CO}_{2}$ se realizó en un reactor. Este dispositivo consta de un recipiente herméticamente cerrado con dos válvulas, una de entrada conectada a una botella de $\mathrm{CO}_{2}$ y otra de salida de gases. Los aerogeles fueron pulverizados y dispersados en agua destilada y, a continuación, sometidos en condiciones de presión atmosférica y temperatura ambiental a un flujo de gas. Los ensayos consisten en dosis-inyección sucesivas de $\mathrm{CO}_{2}$ de 15 minutos cada 24 horas, en las que permanece el reactor en situación de reposo. La formación del carbonato fue constatada mediante difracción de rayos $\mathrm{X}$ y análisis termogravimétrico.

\section{RESULTADOS}

\subsection{Estructura}

El análisis de la textura se realizó mediante técnicas de adsorción de nitrógeno. Las isotermas obtenidas para los aerogeles en estudio se identifican como Tipo IV característica de sólidos mesoporosos (Figura 2). Los ciclos de histéresis que presentan dichas isotermas se pueden identificar como de tipo $\mathrm{H} 2$ que corresponden a una distribución porosa homogénea con un alto grado de interconexión. En todos los casos se observa un primer régimen $\left(0.1<\mathrm{P} / \mathrm{P}_{0}<0.5\right)$ en multicapas hasta llenar los poros más pequeños y que viene seguido $\left(\mathrm{P} / \mathrm{P}_{0}>0.5\right)$ de la condensación capilar en los mesoporos. La aparición de un ciclo de histéresis se debe a que la condensación y la evaporación de los mismos poros se producen a distinta presión. Este efecto se relaciona con la forma e interconexión de los poros. Ciclos de ese tipo se asocia

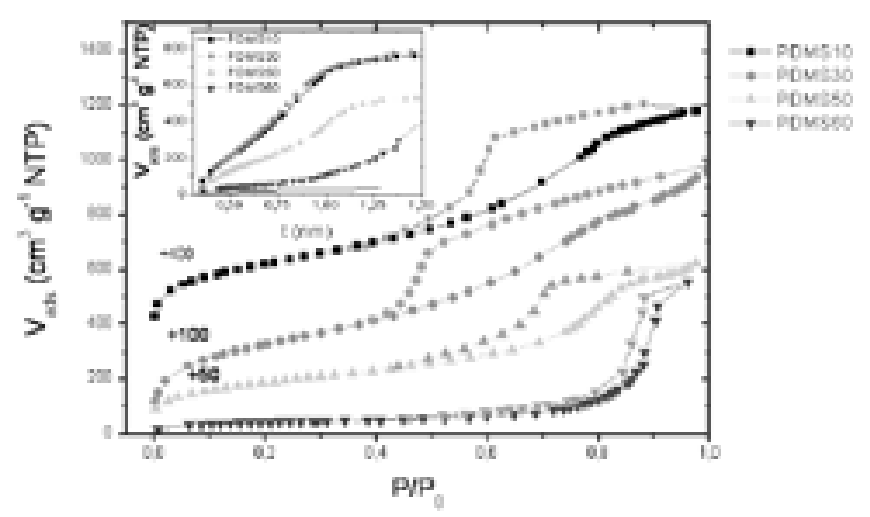

Fig. 2- Isotermas de nitrógeno para los aerogeles indicados, las curvas se han desplazado convenientemente para una mayor claridad. En el interior se muestra una representación V-t que indica la existencia de microporos. Como referencia, la línea continua al pie de la gráfica V-t corresponde a una muestra de sílice no porosa.

a poros formados por partículas casi esféricas empaquetadas o poros tipo tintero. En este sentido se observa un brusco cambio en la forma del ciclo de histéresis para la muestra PDMS60 en donde de la forma del ciclo se infiere la existencia de un entramado de los polímeros formando rendijas. Este resultado viene corroborado por las micrografías de TEM para el aerogel de sílice pura y la muestra PDMS60 (Figura 3), contrastado con la discusión a este respecto de Kruk and Jaroniec (18).

Finalmente, fueron analizadas las representaciones del volumen adsorbido en función del espesor estadístico en la formación de multicapas con el fin de analizar la microporosidad de estos sistemas (t-plots) que están representadas en la gráfica 


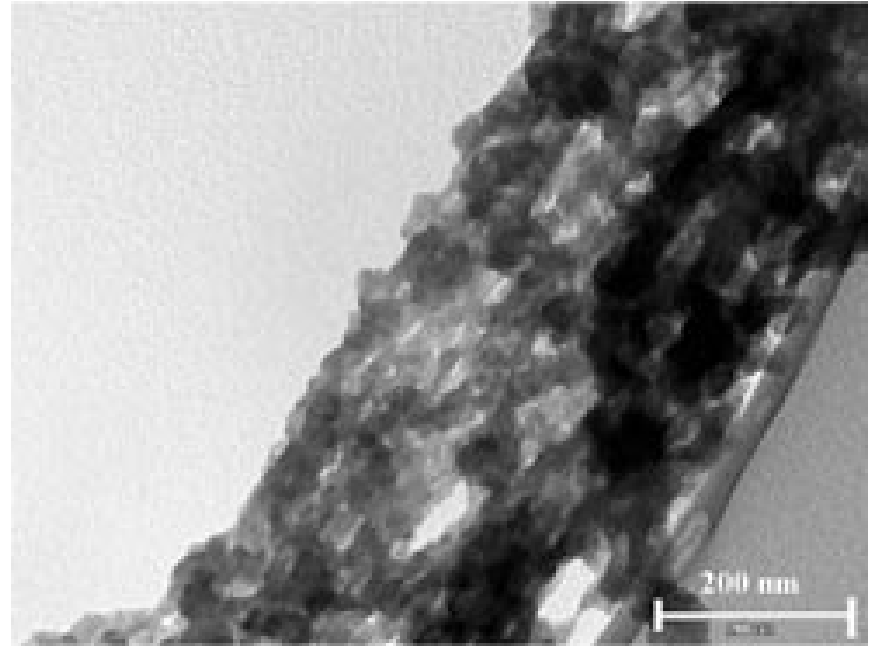

Fig. 3- Microscopía TEM del sono-aerogel híbrido PDMS60 en donde se observa que el entramado de los polímeros forma rendijas.

TABLA I. MUESTRAS PDMSX CON X\% EN PESO DE CONTENIDO EN POLÍMERO CON DOSIS DE ULTRASONIDOS DE $0.64 \mathrm{KJ} / \mathrm{cm}^{3} Y$ SU COMPARACIÓN CON LA MATRIZ DE SÍLICE PURA

\begin{tabular}{c|cc|c|c|cc}
$\mathrm{X} \%$ & $\begin{array}{c}\mathrm{S}_{\mathrm{T}} \\
\left(\mathrm{m}^{2} / \mathrm{g}\right)\end{array}$ & $\begin{array}{c}\mathrm{V}_{\mathrm{T}} \\
\left(\mathrm{cm}^{3} / \mathrm{g}\right)\end{array}$ & $\begin{array}{c}\rho^{\mathrm{a})} \\
\left(\mathrm{g} \mathrm{cm}^{-3}\right)\end{array}$ & $\begin{array}{c}\rho^{\mathrm{b})} \\
\left(\mathrm{g} \mathrm{cm}^{-3}\right)\end{array}$ & $\begin{array}{c}\mathrm{D}_{\mathrm{BH}} \\
(\mathrm{nm})\end{array}$ & $\begin{array}{c}\mathrm{D}_{\mathrm{h}}^{\mathrm{c}} \\
(\mathrm{nm})\end{array}$ \\
\hline 0 & 1441 & 1.474 & 0.519 & 0.598 & 8.2 & 4.1 \\
10 & 1842 & 1.552 & 0.498 & 0.567 & 5.4 & 3.4 \\
20 & 1885 & 1.724 & 0.459 & 0.570 & 6.1 & 3.7 \\
30 & 2010 & 1.642 & 0.477 & 0.638 & 4.1 & 3.3 \\
50 & 1063 & 1.015 & 0.680 & 0.680 & 7.1 & 3.8 \\
60 & 333 & 0.7899 & 0.803 & 0.764 & 15.3 & 9.5
\end{tabular}

insertada en la Figura 2. Un aumento brusco del volumen adsorbido respecto del de una muestra estándar de sílice no porosa es la indicación de dicho carácter microporoso (radio de poro $<2 \mathrm{~nm}$ ). Los resultados de la aplicación de los modelos se ilustran en la Tabla I. Ahí se ha incluido las densidades experimentales medidas directamente pesando los aerogeles cilíndricos de geometría bien definida. Se observa una gran influencia de los parámetros (Tabla I) con el contenido en fase orgánica: el aumento del contenido en polímero provoca un descenso de las variables analizadas junto con un aumento significativo del tamaño del poro, lo que estaría indicando un colapso de la porosidad por la acomodación de las cadenas poliméricas en torno a los cúmulos de sílice. Es de destacar el acuerdo que se observa en la variación de la densidad en función del contenido orgánico entre las medidas geométricas (cilindro monolítico) y las obtenidas a partir de la isoterma.

La evaluación de la microporosidad según el modelo de Dubinin-Radushkevich (Tabla I), indica valores más altos de la superficie específica respecto a los obtenidos por el modelo BET (que muestran un ajuste correcto entre 0.05 y 0.3 de presión relativa) junto con un alto volumen microporoso, lo que confirma la existencia de una importante microporosidad en estos aerogeles. A medida que aumenta el contenido en polímero dicho volumen microporoso disminuye llegando a ser despreciable en el caso de la muestra PDMS60. Este hecho es consecuente con el aumento del tamaño del poro y la caída en la superficie específica.

Los resultados anteriores sugieren una imagen de la estructura de estos aerogeles en la que, para baja concentración en polímero, éste conforma un entramado que se encuentra embebido en la matriz de súlice inorgánica. A medida que aumenta el contenido en polímero, éste pasa a tener un carácter de matriz (no porosa) en la que se encuentran embebidos los cúmulos de la sílice. Así, estos resultados indicarían que mediante secado supercrítico de los aerogeles es posible la obtención de materiales híbridos orgánico/ inorgánico sin que se produzca la degradación térmica de la fase orgánica, como se infiere del análisis TG (15). Tal análisis que se realiza en atmósfera de aire y presión atmosférica, lejos de las condiciones supercríticas del etanol en la autoclave y que influyen en la estabilidad del polímero. Por otro lado, al evitar la ciclidación mediante los ultrasonidos, se favorece la unión entre TEOS y PDMS y, por tanto, la estabilidad de éste último.

\subsection{Comportamiento mecánico}

La curva experimental de esfuerzo-deformación en estos aerogeles se muestra en la Figura 4, cuyo registro se ha realizado hasta producir la fractura del aerogel. Según puede observarse para la muestra sin contenido en polímero tiene un comportamiento frágil, con un módulo de Young de 114 $\mathrm{MPa}$, algo mayor que en el caso de los aerogeles de sílice de baja densidad que tienen en torno a los $10 \mathrm{MPa}$, pero significativamente menor que para la sílice fundida con 73 GPa. Se observa en la curva un comportamiento asintótico que revela la deformación plástica con un módulo de fractura de $8 \mathrm{MPa}(19)$.

Un comportamiento similar se observa para la muestra PDMS10 sin embargo se deforma algo más antes de romperse. El comportamiento elastomérico característico de estos polímeros se pone de manifiesto en el resto de las muestras,

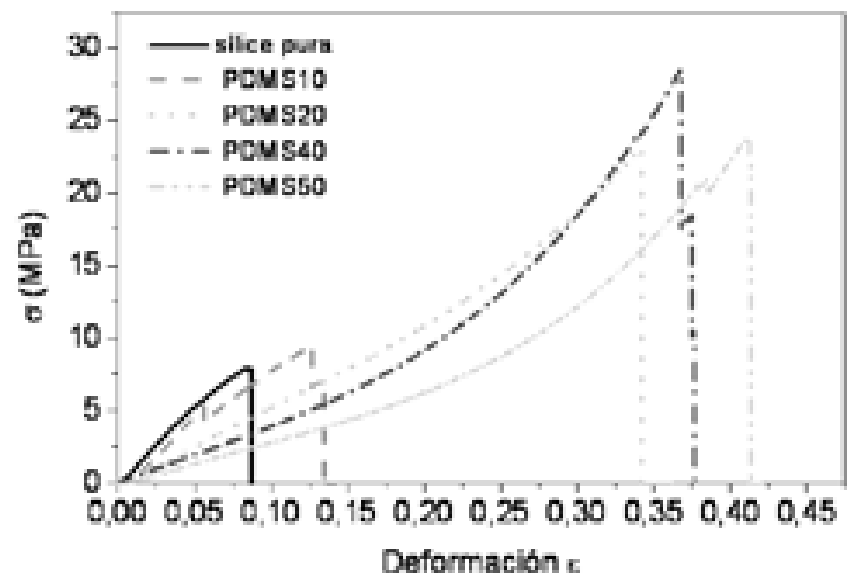

Fig. 4- Curvas tensión-deformación de experimentos de compresión uniaxial.

TABLA II. PARÁMETROS MECÁNICOS MEDIDOS DE LOS RESULTADOS DE LOS ENSAYOS DE COMPRESIÓN UNIAXIAL.

\begin{tabular}{c|cccc|}
$\begin{array}{c}\text { DMS12 } \\
(\%)\end{array}$ & $\begin{array}{c}\text { Tensión de } \\
\text { Compresion } \\
(\mathrm{MPa})\end{array}$ & $\begin{array}{c}\text { Esfuerzo en } \\
\text { fractura } \\
(\%)\end{array}$ & $\begin{array}{c}\text { E 50\% } \\
(\mathrm{MPa})\end{array}$ & $\begin{array}{c}\text { E 90\% } \\
(\mathrm{MPa})\end{array}$ \\
\hline 0 & 8.00 & 8.40 & 113.80 & 101.00 \\
10 & 9.60 & 13.40 & 105.70 & 89.90 \\
20 & 23.10 & 34.70 & 55.10 & 65.60 \\
40 & 28.60 & 36.80 & 41.50 & 78.75 \\
50 & 23.70 & 42.90 & 39.40 & 55.92
\end{tabular}


donde se observa un crecimiento convexo de la curva con un brusco crecimiento en torno al $25 \%$ de la deformación. Resultado que indica una variación del módulo de Young conforme aumenta la carga, según se indica en la Tabla II, en donde a partir de la muestra PDMS20 se produce un aumento del mismo, respecto al módulo medido al $50 \%$ de la carga. Este comportamiento es característico de los elastómeros que al ser comprimidos muestran una mayor rigidez.

Una explicación plausible para este comportamiento es la de suponer una disminución del ángulo de enlace O-Si-O de la red polimérica que de esta forma se comportaría como un muelle presionando sobre los cúmulos de sílice que conforman la red inorgánica.

El carácter viscoelástico de estos materiales lo indica las curvas de relajación en los que se muestra que al cabo de diez minutos la carga necesaria para mantener la deformación es $\sim 1 \%$ mayor que el valor, según se ilustra en la Figura 5.

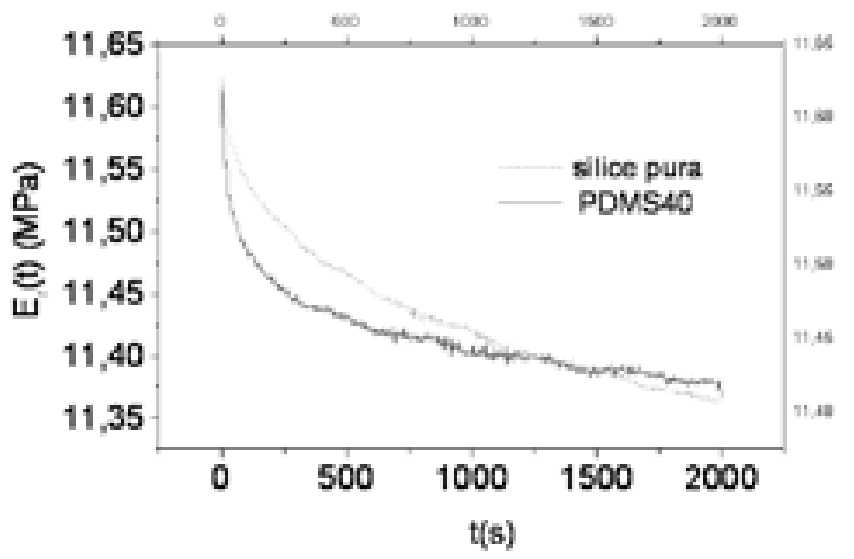

Fig. 5- Experimento de relajación que muestra la carga necesaria en función del tiempo para mantener una deformación fija, revelan el carácter viscoelástico de estos aerogeles.

\section{APLICACIONES DE LAS PARTÍCULAS DE WOLLASTONITA EMBEBIDAS EN AEROGELES}

\subsection{Aerogeles bioactivos.}

Las propiedades físico-químicas que presentan estos aerogeles híbridos orgánico-inorgánico los convierten en materiales con gran interés potencial en aplicaciones bioactivas para reparación de tejidos. $\mathrm{El}$ sistema $\mathrm{SiO}_{2}$-PDMS, como recientemente ha sido comprobado (20) habilita el proceso de biomineralizacion de fosfato cálcico en superficies, lo cual induce a su vez a la integración en los tejidos biológicos. Las especies químicas esenciales que inducen la nucleación heterogénea de la apatita son los grupos silanoles. Más aún, la liberación de iones $\mathrm{Ca}^{2+}$ desde la superficie del material, ayuda a la deposición de la apatita ya que aumenta el grado de supersaturación del plasma sanguíneo en relación con la apatita. Con estas premisas, era de esperar que la inclusión del ión calcio en nuestros aerogeles los convierta en materiales que combinan la flexibilidad, debida a su comportamiento elastomérico, con la bioactividad.
La microscopía electrónica de barrido realizada a las superficies de fractura de los aerogeles, permite apreciar una alta dispersión de las partículas, encontrándose éstas homogéneamente distribuidas en el aerogel, con agregados de tamaños comprendidos entre 1 y $5 \mu \mathrm{m}$ de diámetro.

Los aerogeles $\mathrm{SiO}_{2}-\mathrm{PDMS} / \mathrm{CaSiO}_{3}$ resultantes son materiales compuestos, cuyas propiedades mecánicas resultan modificadas respecto a las de los aerogeles de partida. Los ensayos de compresión uniaxial llevados a cabo sobre cilindros de $8 \mathrm{~mm}$ de diámetro y $15 \mathrm{~mm}$ de altura, revelan importantes diferencias entre aerogeles con y sin wollastonita. La diferencia más notable es, aparte de la disminución de la resistencia a la rotura, desde 18 a $8 \mathrm{MPa}$, en algunas de las muestras, es la disminución de su módulo de elasticidad de 100 a $50 \mathrm{MPa}$, encontrándonos dentro del intervalo de los valores de resistencia mecánica y módulo de Young propios del hueso esponjoso (5 a $10 \mathrm{MPa}$ y 50 a $100 \mathrm{MPa}$, respectivamente) (21 22,23, 24) (Figura 6).

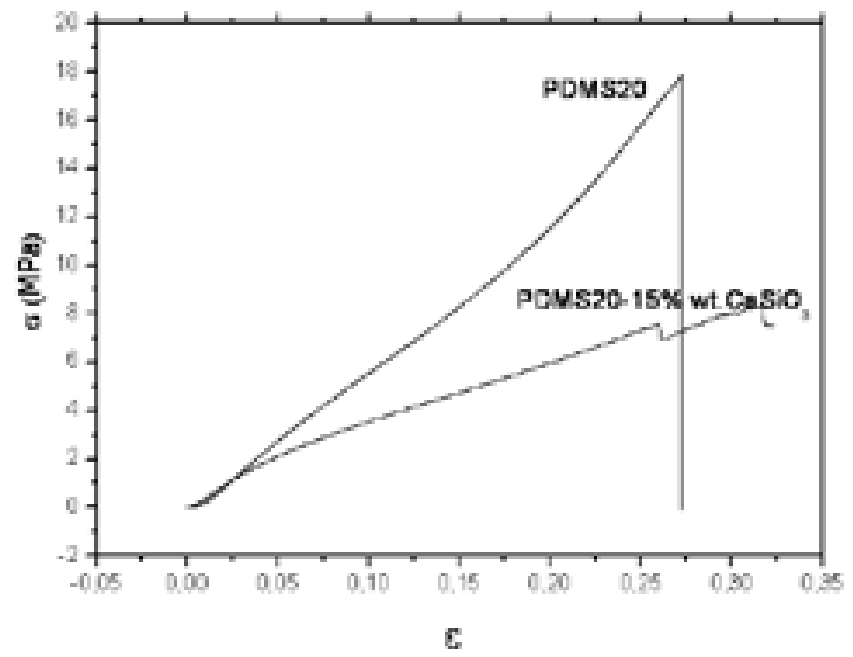

Fig. 6- Curvas carga-deformación de los aerogeles híbridos PDMS 20 y PDMS20/15\% en peso de $\mathrm{CaSiO}_{3}$.

El resultado es la aparición de esférulas de hidroxiapatita de tamaño regular, $(5 \mu \mathrm{m})$, desigualmente distribuidas sobre la superficie de las pastillas tratadas a $500{ }^{\circ} \mathrm{C}$, al cabo de una semana de inmersión en SBF (Figura 7, y Figura 8). La formación de apatita sobre la wollastonita tratada a $1000{ }^{\circ} \mathrm{C}$ tiene lugar de forma más homogénea, sobre toda la superficie, no observándose empaquetamientos en forma de esferas tras 8 días de inmersión en SBF (Figura 8 a), a diferencia del caso anterior. Estas estructuras de esferas empaquetadas sí las encontramos en las muestras tratadas a $1000^{\circ} \mathrm{C}$ tras 15 días en SBF (Figura 8 b).

\subsection{Aerogeles fijadores de $\mathrm{CO}_{2}$}

Las propiedades físico-químicas ya citadas de los aerogeles, los convierten también en sustratos adecuados para tecnologías con aplicaciones medioambientales. Entre estas destacan la captura y fijación de gases atmosféricos, fundamentalmente $\mathrm{CO}_{2}$. Estas aplicaciones se deducen de la observación de la Figura 9. En ella se muestran los diagramas de rayos $\mathrm{X}$ correspondientes a tres situaciones temporales diferentes, observándose la evolución del polvo sintético de 

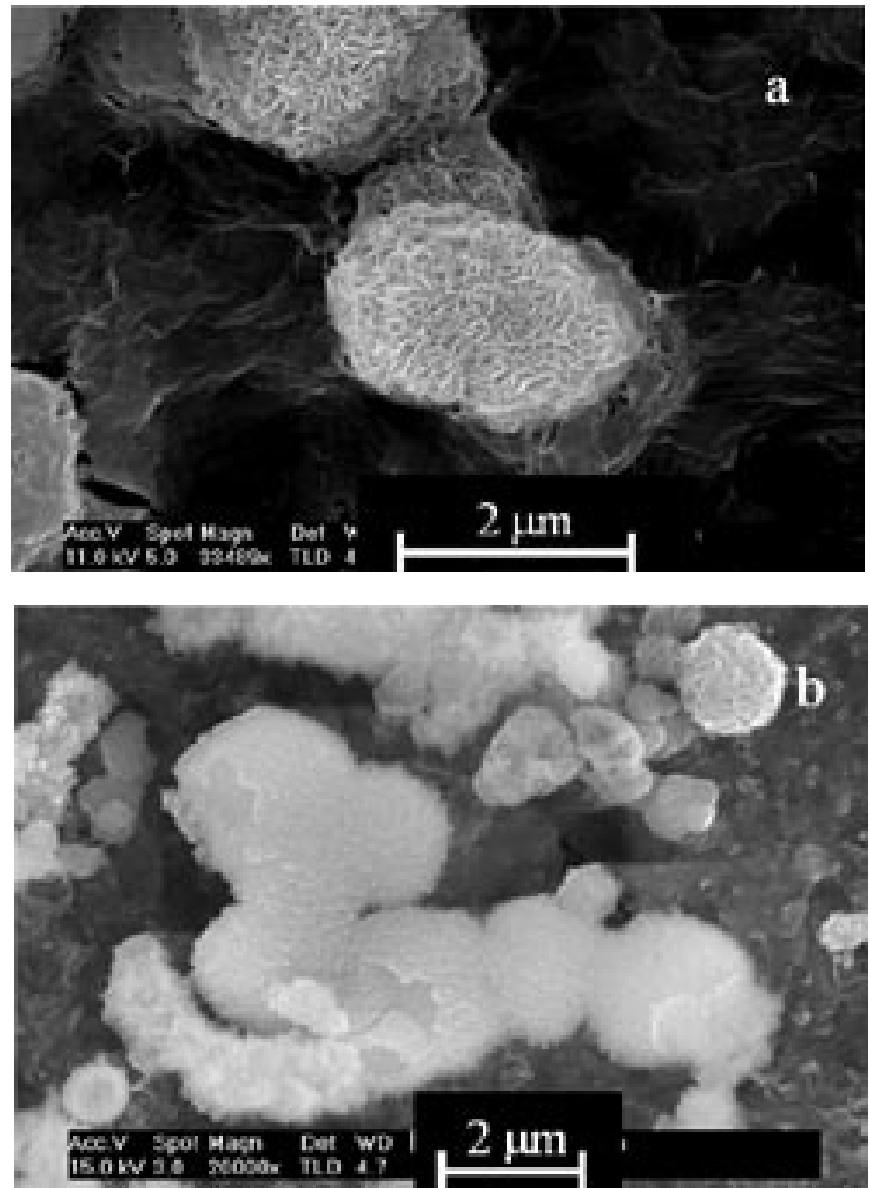

Fig. 7- Micrografías de microscopía electrónica de barrido de partículas de wollastonita calcinada a $500^{\circ} \mathrm{C}$ tras 8 días (a) y 15 días (b) inmergidas en SBF.

wollastonita después de haber inyectado en tres ocasiones un flujo continuo de $\mathrm{CO}_{2}$, durante 15 minutos. Esto permite evaluar la transformación wollastonita-calcita a partir de la identificación de ambas fases. Los resultados contrastan favorablemente con aquellos obtenidos por otros autores (12) sobre muestras naturales y donde la reacción de carbonatación, aunque favorecida termodinámicamente, ocurre de forma muy lenta mediante un proceso de disolución del $\mathrm{Ca}$ (II) y posterior formación del carbonato como indicador fiable de la verificación de la reacción de fijación del $\mathrm{CO}_{2}$.

Igualmente concluyentes son los resultados que muestran los diagramas de rayos $X$ de la Figura 10, (abajo) que corresponden a aerogeles con la fase activa incorporada al $9 \%$ y al $26 \%$ en peso. En la primera de las muestras se observa un componente mayoritariamente amorfo debido a la sílice de la matriz y a la formada de la descomposición de wollastonita, como un producto de la reacción ocurrida en la que también se ha formado carbonato de calcio. Esta muestra con escaso contenido de Ca (II), y después de un único ensayo, verifica que la reacción ha tenido lugar por la presencia de picos de difracción correspondientes al carbonato.

Esto mismo se confirma en la Figura 10, (arriba) en la que se aprecia una mayor intensidad en los picos de difracción del carbonato, lo que se interpreta como mayor capacidad de fijación al incrementar el contenido de polvo de wollastonita en la matriz de aerogel. En ambos casos las condiciones experimentales son idénticas. La verificación de la reacción de
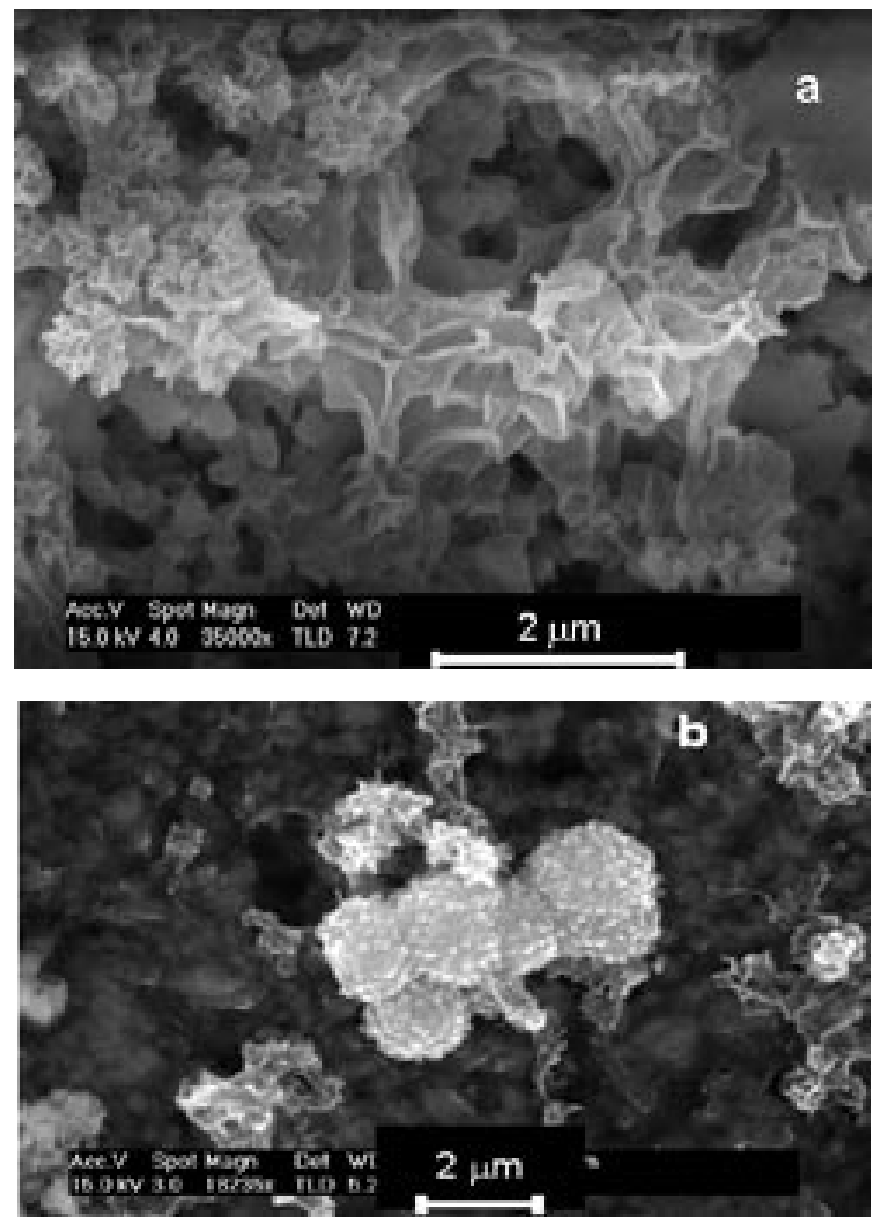

Fig. 8- Micrografías de microscopía electrónica de barrido de una pastilla de wollastonita tratada a $1000^{\circ} \mathrm{C}$ después de 8 días (a) y 15 (b) días de ser sumergida en SBF.

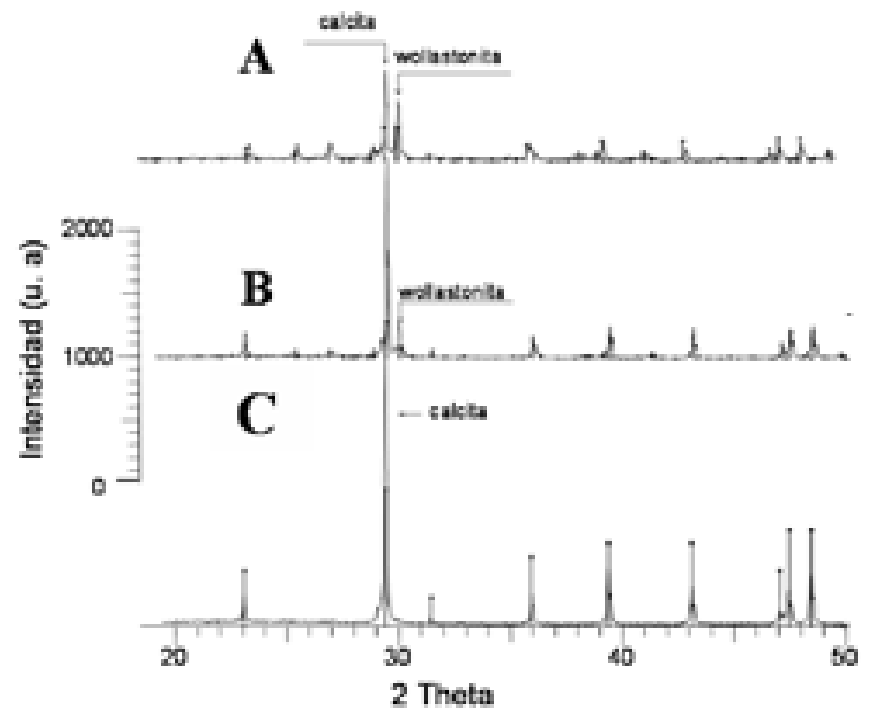

Fig. 9- Diagramas de Rayos $X$ de la transformación polvo de wollastonita-calcita. A) primera dosis-inyección de $\mathrm{CO}_{2}$. B) Segunda dosisinyección y C) Tercera dosis-inyección.

fijación del $\mathrm{CO}_{2}$ queda de manifiesto en el resultado mostrado en la Figura 11, que corresponde al análisis termogravimétrico. En ella se puede apreciar el pico de descomposición del 


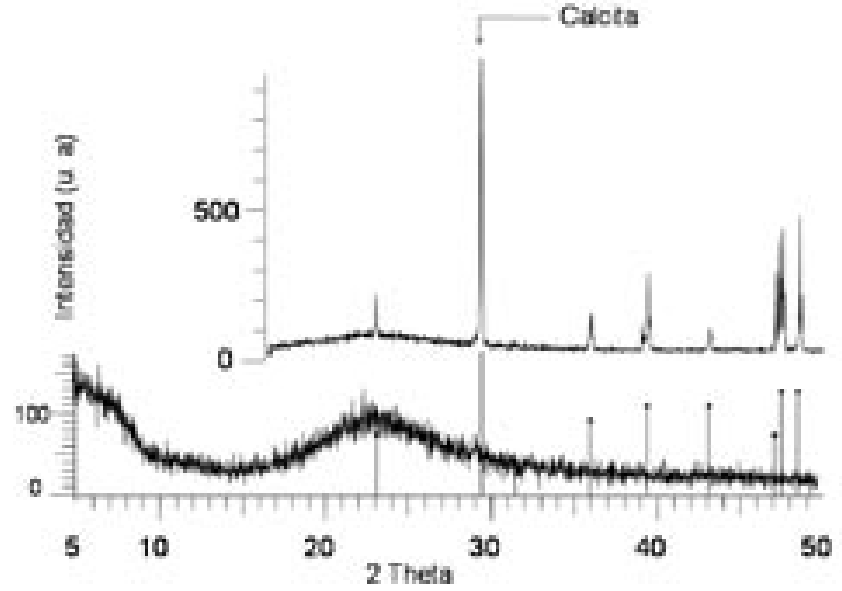

Fig. 10- Patrones de difracción de las muestra aerogeles conteniendo, $9 \% \mathrm{CaO}$ (abajo) y $26 \% \mathrm{CaO}$ (arriba) en peso.

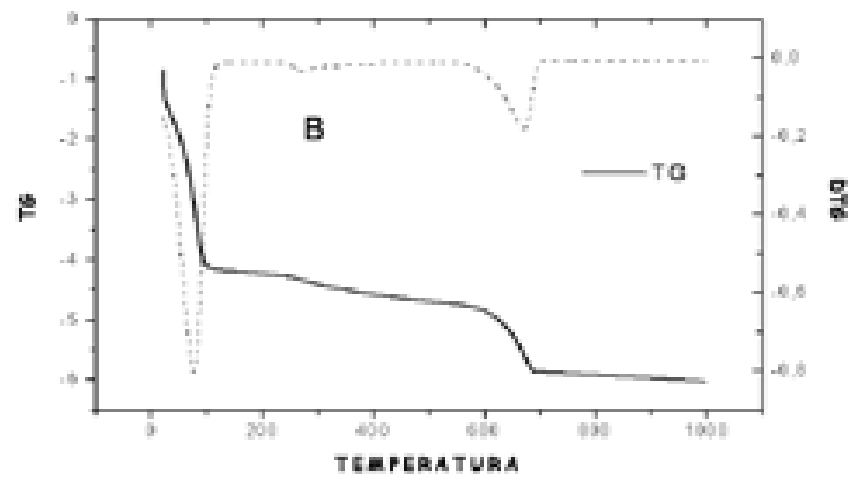

Fig. 11- Curvas TG y DTG de la muestra Aerogel (26\% CaO en peso).

carbonato en el rango entre $535-650{ }^{\circ} \mathrm{C}$, que previamente ha sido chequeado para una muestra pura de carbonato en atmósfera de oxígeno.

\section{CONCLUSIÓN}

La combinación de las propiedades que son propias de los sonogeles como la alta superficie específica y densidad cuando contienen una fase orgánica los convierte en materiales adecuados para soportar una fase activa para su aplicación en campos de naturaleza dispar. Así, hemos preparado materiales compuestos sono-aerogel híbrido/ wollastonita que se muestran muy bioactivos y son capaces de fijar $\mathrm{CO}_{2}$. En el primer caso obtenemos materiales que, por su bioactividad y comportamiento mecánico en compresión, son válidos para continuar su estudio como implantes óseos y, en el segundo caso, gracias a su eficaz reactividad con $\mathrm{CO}_{2}$, como controladores de la contaminación ambiental.

\section{BIBLIOGRAFÍA}

1. S.S. Kistler, “Coherent Expanded Aerogels”, J. Phys. Chem. 36, 52 (1932).

2. http://www.guinnessworldrecords.com/content_pages/recordd asp? recordid $=47186$.

http://www.llnl.gov/str/October03/NewsOctober03.html.

3. J. Fricke and T. Tillotson, "Aerogels: production, characterization, and applications", Thin Solid Films 297, 212-223 (1997).

4. N. Hüsing and U. Schubert, “Aerogels-Airy Materials: Chemistry, Structure, and Properties", Angew. Chem. Int. Ed. 37, 22-45 (1998).

5. L. Esquivias y J. Zarzycki "Sonogels: An Alternative Method in Sol-Gel Processing" en Ultrastructure Processing of Advanced Ceramics, Ed. John Wiley \& Sons, Inc, (1988) pp. 255- 270.

6. T. Kokubo, H. Kushitani, S. Sakka, T. Kitsugi and T. Yamamuro, "Solutions able to reproduce in vivo surface-structure changes in bioactive glassceramic A-W"', J. Biomed. Mater. Res.24 (1990) 721-726.

7. G. Vargas Gutiérrez, J. Méndez Novell, L. López Ojeda, P. N. de Aza S. de Aza, "Cementos odontológicos a base de Poli (ácido acrílico) y wollastonita", Bol. Soc. Esp. Ceram. V., 44 [2] 89-94 (2005).

8. P.N. de Aza, A. H. Aza, S. de Aza, "Crystalline Bioceramic Materials”, Bol. Soc. Esp. Ceram. V., 44 [3] 89-94 (2005).

9. B. Hosticka, P.M. Norris, J.S. Brenizer, and C.E. Daitch, “Gas flow through aerogels" J. Non-Cryst. Solids. 225 (1998) 293-297.

10. O.Leal, C. Bolívar, C. Ovalles, J.J. García, Y. Espidel, “Reversible adsorption of carbon dioxide on amine surface-bonded silica gel", Inorg. Chim. Acta 240 (1995) 183-189.

11. M.S. Ahmed and Y.A. Attia, "Multi-metal oxide aerogel for capture of pollution gases from air", App. Thermal Eng. 18, (1998).787-797.

12. J.C-S Wu, J-D, Sheen, S-Y Chen, Y-Ch Fan "Feasitrility of $\mathrm{CO}_{2}$ fixation via artificiall rock weathering". Ind. Eng Chem. Res. 40 (2001) 3902.

13. L. Esquivias, V. Morales-Flórez, M. Piñero, N. de la Rosa-Fox, J. Ramírez, J. González-Calbet, A. Salinas, M. Vallet-Regí, “Bioactive organic-inorganic hybrid aerogels" MRS Proceedings 847, (2005) EE12.1.1 - EE12.1.6.

14. C. Jiménez-Solís, C. Barrera-Solano, A. Pérez-Moreno y L. Esquivias, "Influencia de la naturaleza del precursor en la morfología de polvos YSZ "Bol. Soc. Ceram. V., [36] 405-411 (1997).

15. N. de la Rosa-Fox, L. Esquivias and M. Piñero, “Organic-Inorganic Hybrid Materials from Sonogels" en Handbook of Organic-Inorganic Hybrid Materials and Nanocomposites 1 (2003) 241-270, (Ed. S.H. Nalwa). American Scientific Publishers.

16. M.M. Dubinin and L.V. Radushkevich, "Some new tour parameter models for moisture sorption isotherms". Dokl. Akad. Nauk SSSR 55 (1947) 327.

17. E.P. Barret, L.G. Joyner and P.P. Halenda, "The determination of pore volume anda rea distributions in porous substances, I: Computations from nitrogen isotherms", J. Am. Chem. Soc. 73 (1951) 373.

18. M. Kruk and M. Jaroniec, ., "Gas adsorption characterization of ordered organic-inorganic nanocomposites materials" Chem. Mater. 13(10) (2001) 3169-3183.

19. M. Piñero, V. Morales-Flórez, N. de la Rosa-Fox and L. Esquivias, "Mechanical properties of silica hybrid aerogels", Bol. Soc. Esp. Ceram. V., 44 [5] 291 (2005).

20. K-Y. Lee, Y.-H. Lee, H.-M. Kim, M.-Y. Koh, S.-H. Ahn, H.-K. Lee, "Synthetic Model Of A Bioactive Functionally Graded Nano-Hybrid In Silica-Polydimethylsiloxane System", Current Appied Physics 5 (2005) 453-457.

21. Suso-Vergara, S., Tesis doctoral, "Estudio comparativo de la utilidad de los cementos y vidrios en fosfato de calcio como sustitutivos óseos en defectos cavitarios experimentales", Universitat de Barcelona, 2004.

22. Comin M., Peris J.L., Prat, J.M., Dejoz, J.R., Vera, P.M., Hoyos, J.V.: "Biomecánica de la fractura ósea y técnicas de reparación", 1999, Instituto de Biomecánica de Valencia, Valencia Ed. Martín.

23. Proubasta, I., Gil, J., Planell, J.A., "Fundamentos de Biomecánica y Biomateriales", 1999, Madrid, Ed. Ergon.

24. Cowin, S.C., "Bone Mechanics", Boca Raton, Florida, CRC Press.

Recibido: 20.01.06

Aceptado: 23.10 .06 